PSICOLOGÍA

IBEROAMERICANA
Psicología Iberoamericana ISSN: 1405-0943

revista.psicologia@ibero.mx

Universidad Iberoamericana, Ciudad de México México

\title{
La violencia contra las mujeres en los países en desarrollo: México y la crisis de migrantes
}

Gordon, Sarah Frances

La violencia contra las mujeres en los países en desarrollo: México y la crisis de migrantes

Psicología Iberoamericana, vol. 26, núm. 1, 2018

Universidad Iberoamericana, Ciudad de México, México

Disponible en: http://www.redalyc.org/articulo.oa?id=133959553001 


\title{
La violencia contra las mujeres en los países en desarrollo: México y la crisis de migrantes
}

Violence against women in the developing world: Mexico and the migrant crisis

\author{
Sarah Frances Gordon sarah.gordon@ibero.mx \\ Universidad Iberoamericana, México
}

La violencia contra las mujeres es una epidemia mundial que afecta a 1 de cada 3 mujeres en su vida (Banco Mundial, 2018). La investigación global sugiere que hasta un $38 \%$ de los feminicidios son cometidos por sus parejas (oms, 2017). La violencia contra las mujeres y la seguridad pública son algunos de los desafíos que enfrenta México en este momento (acnudh, 2018). El Instituto Nacional de Estadística y Geografía (inegi), estima que el $66.1 \%$ de las niñas y mujeres de 15 años o más han sufrido violencia de género al menos una vez en su vida y que el $43.5 \%$ de las mujeres han sufrido violencia de género cometida por sus parejas (Amnistía Internacional, 2018). El femicidio es un problema nacional en México y al menos 400 mujeres han sido brutalmente asesinadas en la última década en Ciudad Juárez (Joseph, 2017; oms, 2012). Sin embargo, México sigue plagado por la falta de datos fiables sobre la violencia contra las mujeres, lo que hace que sea difícil estimar con exactitud el alcance de esta epidemia.

La violencia contra las mujeres parece estar arraigada culturalmente en México. Como se puede ver en la investigación realizada a inmigrantes mexicanos por Bauer, Rodríguez, Quiroga, y Flores-Ortiz (2000), las mujeres le dan mayor prioridad a mantener sus matrimonios intactos que a su propia seguridad, a menudo tolerando abuso de sus parejas basado en sus creencias en la santidad del matrimonio y la importancia de la familia. Por otra parte, la prevalencía de la violencia contra las mujeres en México puede ser vista como un resultado de la crisis que actualmente vive el país, el gobierno, la seguridad interna, el tráfico de drogas, así como la cultura del machismo en el país (Ertürk, 2006; acnudh, 2018; Olivera, 2006) en la que los hombres exageran los aspectos violentos, autoritarios y agresivos de la identidad masculina, y se puede ver en la desigualdad de género socialmente arraigada y las estructuras patriarcales y sexistas en México (acnudh, 2018; Olivera, 2006). La cultura machista en México relega a las mujeres a roles de subordinación, donde se espera que ellas se definan en relación con sus maridos y las responsabilidades domésticas, negándoseles una existencia independiente (Ertürk, 2006). Esto hace que les sea difícil combatir el abuso y acceder a canales de apoyo. También crea un entorno 
donde la violencia contra las mujeres puede florecer. Estos factores se ven agravados por otros factores de estrés en el país, como el desempleo, la pobreza, la polarización social y la incapacidad del gobierno y el sistema legal para responder eficazmente a la delincuencia, por lo que en México las mujeres son particularmente susceptibles a los altos niveles de violencia (Ertürk, 2006; Olivera, 2006).

Las mujeres indígenas en México también son objeto de violencia debido a las jerarquías patriarcales tradicionales de las comunidades indígenas, los estereotipos y la discriminación (Ertürk, 2006). La investigación indica que las autoridades de las comunidades indígenas utilizan las normas habituales y sociales para normalizar la violencia doméstica, que consolida aún más la marginación de estas mujeres en México (Mejía Flores, 2006).

Adicionalmente, las situaciones de conflicto y desplazamiento, como la actual crisis migratoria en Centroamérica y América Latina, pueden exacerbar la violencia contra las mujeres existente en la región. Las mujeres y niñas migrantes suelen entrar en México en busca de mejores oportunidades o en tránsito a los ee.uu (oms, 2017). A lo largo de la ruta a través de México, la violencia contra las mujeres representa un riesgo, principalmente en la frontera sur México con Guatemala, donde la situación es particularmente volátil (Cuffe, 2018; Ertürk, 2006). Por otra parte, la naturaleza de los patrones de migración hace que las mujeres y niñas migrantes sean especialmente vulnerables a la explotación, violencia y tráfico de personas (Ertürk, 2006; acnudh, 2018). La vulnerabilidad de las mujeres indocumentadas en México se ve incrementada por las leyes locales que prohíben el acceso a las autoridades estatales. De igual manera la Ley General de Población prohíbe a las autoridades procesar cualquier solicitud de una persona que no sea capaz de demostrar su estatus legal en México (Ertürk, 2006). Mientras que las tensiones sobre la inmigración continúan intensificando en la región, los ministros de asuntos exteriores esperan que el nuevo plan de desarrollo integral, que promete promover el desarrollo en Centroamérica y México, ayude a revitalizar la región y abordar las causas estructurales de la crisis migratoria (eclac, 2018).

Abordar el problema de la violencia contra la mujer en México requiere un enfoque multifacético y complejo, que también considera los desafíos sociales y políticos que enfrenta el país. Las formas más efectivas para reducir la violencia contra las mujeres incluyen programas educativos destinados a desafiar los roles de género, así como iniciativas que aborden las normas sociales que refuerzan la aceptación de la violencia en la sociedad. Si bien se han aprobado e implementado leyes que procesen a los autores de femicidio y otras formas de violencia contra la mujer en México, éstas hacen poco para hacer frente a la cultura machista, que es responsable de la perpetuación de la desigualdad de género y la subordinación de las mujeres (Joseph, 2017). Sólo una vez que las creencias ideológicas patriarcales, arraigadas en la cultura machista se desmantelen, México será capaz de librarse de la violencia contra las mujeres. 
El campo de la psicología desempeña un papel importante en el abordaje de los retos sociales que enfrenta México, concretamente la violencia contra las mujeres y la crisis migratoria. Los psicólogos están a la vanguardia de estos problemas y necesitan ser conscientes de las fuerzas impulsoras detrás de estos problemas sociales. Por lo tanto, como profesionales, es importante que nos desafiemos a nosotros mismos y abordemos cualquier idea preconcebida que tengamos sobre la violencia contra las mujeres y la crisis migratoria para que seamos más capaces de servir a nuestra communidad.

\section{REFERENCIAS}

acnudh. (2018). acnudh | Comité para la Eliminación de la Discriminación contra la Mujer examina el informe de México. Recuperado de https://www.ohchr.org/EN/NewsEvents/Pages/DisplayNews. aspx? NewsID $=23344 \&$ LangID $=\mathrm{E}$

Amnistía Internacional. (2018). México 2017/2018 | Amnistía Internacional. Recuperado de https:// www.amnesty.org/en/countries/europe-andcentral-asia/kazakhstan/report-kazakhstan/

El Banco Mundial. (2018). Gender-based violence (Violence against women and girls). Recuperado de http://www.worldbank.org/en/topic/socialdevelop ment/brief/violence-against-women-and-girls

Bauer, H. M., Rodriguez, M. A., Quiroga, S. S., \& Flores-Ortiz, Y. G. (2000). Barriers to health care for abused Latina and Asian immigrant women. Journal of Health Care for the Poor and Underserved, 11(1), 33-44. http:/ /dx.doi.org/10.1353/hpu.2010.0590

BBC News (2 de enero de 2019). US fires tear gas at migrants at Mexico border crossing. BBC News. Recuperado de https://www.bbc.com/news/worldus-canada-46739126

Cuffe, S. (21 de octubre de 2018). Honduran women find safety in numbers in migrant caravan. Aljazeera. Recuperado de https://www.aljazeera.com/news/2018/10/honduran-women-find-sa fety-numbers-migrant-caravan-181021185658508.html

Comisión Económica para América Latina y el Caribe (elcac). (10 de diciembre de 2018). Foreign ministers of El Salvador, Guatemala, Honduras and Mexico presented Comprehensive Development Plan in the framework of the Conference on the Global Compact for Migration [Press Release]. Recuperado de https://www.cepal.org/en/pressreleases/foreign-minister $s$-salvador-guatemala-honduras-and-mexico-presented-comprehensive

Ertürk, Y. (2006). Integration of the human rights of women and the gender perspective: Violence against women. United Nations Economic and Social Council (Vol. 70793). https://doi.org/10.1017/S0020818300006640

Joseph, J. (2017). Victims of femicide in Latin America: Legal and criminal justice responses. TEMIDA, 20(1), 3-21. https://doi.org/10.2298/TEM 1701003J

Kinosian, S., \& Partlow, J. (26 de noviembre de 2018). "The situation keeps getting worse": Unrest at U.S-Mexico border creates new tension over migrant caravan. The Washington Post. Recuperado de https://www.washi ngtonpost.com/world/the_americas/mexican-police-deploy-around-car 
avan-migrants-after-day-of-chaos-at-border/2018/11/26/7c500364-f10 9-11e8-99c2-cfca6fcf610c_story.html?noredirect $=$ on\&utm_term $=.437$ $801113 \mathrm{fb} 4$

Mejía Flores, S. (2006). Mujer indígena y violencia: Entre esencialismos y racismos. Revista México Indígena, 5.

Montes, J., Mazars, N., Cohen, N. S., \& Siegal, E. (4 de diecembre de 2018). Fleeing poverty and violence, Central American women explain why they join caravans. Wall Street Journal. Recuperado de https://www.wsj.com/articles/fleeing-poverty-and-violence-central-a merican-women-explain-why-they-join-caravans-1543919400

Olivera, M. (2006). Violencia femicida. Latin American Perspectives, 33(2), 104-114. https://doi.org/10.1017/S0020818300006640

Organización Mundial de la Salud (oms). (2012). Understanding and addressing violence against women: Femicide. Recuperado de http://www.who.int/ reproductivehealth/publications/violence/rhr_11_35/en/

Organización Mundial de la Salud (oms). (2017). Violence against women. Recuperado de http://www.who.int/news-room/fact-sheets/detail/viole nce-against-women 